\title{
Impacto de los subsidios al agua en los hogares pobres de la Ciudad de México
}

\author{
Impact of Water Subsidies on Poor Homes in Mexico City
}

Daniel A. Revollo-Fernández, Lilia Rodríguez-Tapia y Jorge A. Morales-Novelo*

Resumen: En el presente estudio se realiza un análisis de incidencia del subsidio al agua domiciliaria para los hogares de la Ciudad de México. Para ello se identifican los beneficiarios de las transferencias con la encuesta Hábitos de Consumo, Servicio y Calidad del Agua por Hogar en la Ciudad de México 2011, apoyada en el Censo de Población y Vivienda 2010 y la Encuesta Nacional de Ingresos y Gastos de los Hogares 2010, 2012, 2014. Por medio de un análisis de incidencia de subsidios, y el cálculo tanto del indicador del desempeño de términos de focalización y el coeficiente de concentración, los resultados indican que el subsidio no se concentra en los sectores más pobres. Considerados conjuntamente, 20 por ciento de los hogares con mayores ingresos económicos recibe alrededor de 45 por ciento del subsidio, mientras que 20 por ciento de los hogares con menores ingresos económicos concentran poco menos de 12 por ciento de los mismos (subsidio regresivo), lo que pone en evidencia la inequidad en su distribución y la dificultad de focalizarlos hacia

*Daniel A. Revollo-Fernández está adscrito al área de Crecimiento y Medio Ambiente, Departamento de Economía, Universidad Autónoma Metropolitana, Unidad Azcapotzalco.Tel: (+5255) 53189427. Correo-e: darevollof@conacyt.mx, drevollofer@gmail.com. orciD: http://orcid.org/0000-0001-6350-0924. Lilia Rodríguez-Tapia está adscrita al Departamento de Economía, Universidad Autónoma Metropolitana, Unidad Azcapotzalco. Tel: (+5255) 5318 9427. Correo-e: 1rt091056@gmail.com. orcID: https://orcid.org/0000-00021456-999X. Jorge A. Morales-Novelo está adscrito al Departamento de Economía, Universidad Autónoma Metropolitana. Tel: (+5255) 5318 9427. Correo-e: jamn8647@gmail.com. orciD: https://orcid.org/00000002-9143-2452. Universidad Autónoma Metropolitana, Av. San Pablo 180, colonia Reynosa Tamaulipas, Azcapotzalco, Ciudad de México, 02200.

El presente artículo fue elaborado con el apoyo financiero del Consejo Nacional de Ciencia y Tecnología (Conacyt) en el marco del proyecto Hacia una Gestión Integral del Agua por Cuenca Hidrográfica: Un Análisis de Disponibilidad y Usos, Fondo Problemas Nacionales (proyecto núm. 120-2015) y con el respaldo al Proyecto Modelo Hídrico y Económico de la Cuenca del Valle de México en el marco del Programa Cátedras Conacyt. La encuesta fue levantada con recursos del Instituto de Ciencia y Tecnología del Distrito Federal (ICyTDF) en el marco del proyecto Calidad y Disminución de la Demanda de Agua en la Ciudad de México, clave 13311378, 2011.

Artículo recibido el 22 de noviembre de 2016 y aceptado para su publicación el 29 de noviembre de 2017. 
los hogares que más los necesitan. Estos resultados dejan clara la necesidad de revisar esta política de subsidios, no sólo a nivel local, con el desafío que implica que en el proceso no se afecte a los sectores más vulnerables y por ende llegar a mejorar el bienestar de toda la sociedad.

Palabras clave: subsidios, incidencia, inequidad, pobreza, agua domiciliaria.

Abstract: In the present study an analysis of incidence of the subsidy on household water for households in Mexico City is made. For this purpose the transfer recipients are identified using the survey consumer habits, Service and Water Quality Home in Mexico City 2011, supported by the Census of Population and Housing 2010 and the National Survey of Income and Expenditure Survey 2010, 2012, 2014. Through an analysis of incidence of subsidies, and the calculation of both performance indicator terms of focus and concentration ratio, the results indicate that the subsidy is not concentrated in the poorest sectors. Taken together 20 per cent of households with higher incomes receive around 45 per cent of the subsidy, while 20 per cent of households with lower incomes concentrated just under 12 per cent of them (regressive subsidy), revealing the inequity in distribution and focus them difficult to households who need them most. These results make clear the need to look over this policy of subsidies, not only locally, with the challenge that the process will not affect the most vulnerable and thus get to improve the welfare of the whole society.

Keywords: subsidies, incidence, inequality, poverty, household water.

\section{INTRODUCCIÓN}

nivel mundial, en particular en los países menos desarrollados, los organismos - Loperadores de agua potable, saneamiento básico y electricidad, otorgan subsidios al consumo de los hogares que demandan sus servicios (Komives et al., 2005). Uno de los principales argumentos para ofrecer dichos subsidios es que mejoran el bienestar socioeconómico de los hogares, especialmente de los más pobres, al facilitar el acceso a este tipo de servicios y mejorar la redistribución de los recursos gubernamentales (Barde y Lehmann, 2014). A contrapunto, a menudo se considera que el subsidio a los hogares genera ineficiencia en el uso de los recursos y desequilibrios financieros en los organismos operadores (Whittington, et al., 2015). En ese marco de tensión, el impacto de los subsidios siempre ha generado controversias en ambos sentidos (Komives et al., 2005).

Sin embargo, la magnitud de los subsidios que se otorgan a los usuarios de servicios públicos puede representar un costo de oportunidad para el gasto público del Estado y para los costos de operación y mantenimiento de las empresas que prestan los servicios de agua. Por ejemplo, Kochhar et al. (2015) estiman que la cantidad de subsidios al agua potable a nivel mundial para el año $2012 \mathrm{impli-}$ caba un valor aproximado de 456 billones de dólares, esto es, 0.6 por ciento del 
producto interno bruto mundial (PIB). Por otra parte, aumentar el acceso al agua de sectores vulnerables, como los hogares con bajos ingresos, contribuye a la reducción de la pobreza y la mejora de su bienestar.

Numerosos estudios han demostrado que el mejoramiento y acceso a los servicios de agua y saneamiento incrementan el bienestar de los hogares más pobres, al reducir la mortalidad general, las enfermedades y padecimientos como la desnutrición, el raquitismo y la menor productividad laboral, entre otros (Listori, 1996; Kelly et al., 2003; Galiani et al., 2005). Existe una variedad de estudios donde se analiza la incidencia de los subsidios en el sector de agua potable y saneamiento básico, centrados en la estructura tarifaria y su impacto sobre los hogares pobres (Komives et al., 2005, 2006, 2007; Bardasi y Wodon, 2008; Banerjee et al., 2010). Los estudios se concentran en examinar la magnitud del subsidio (Walker et al., 2000; Foster y Araujo, 2004), comparar su eficiencia en la forma de tarifas sociales (Foster, 2004; Barde y Lehmann, 2014) y verificar su repercusión sobre el número de conexiones (Ángel-Urdinola y Wodon, 2012; Whittington, et al., 2015), así como al consumo de agua que por lo general resulta inequitativo al comparar su distribución entre los diferentes grupos de la sociedad (Kochhar et al., 2015).

En México, de acuerdo con la Ley de Aguas Nacionales, los gobiernos locales son responsables del suministro de agua de uso público urbano (LNA, 2016). El gobierno de la Ciudad de México (CDMx) adoptó la modalidad de la provisión pública del servicio mediante un único organismo operador, el Sistema de Aguas de la Ciudad de México (Sacmex), que es el organismo público responsable del suministro de agua a los residentes de la ciudad.

El principal costo de suministro del agua es la red de tuberías; una vez que ha sido instalada, los costos adicionales son relativamente pequeños, y éste es el argumento de algunos gobiernos para justificar el monopolio natural del Estado (Stiglitz, 2000), en este caso a través de la empresa pública Sacmex.

Cuando un gobierno local suministra un bien privado como el agua, cada $\mathrm{m}^{3}$ de agua que se consume tiene un costo de producción, incluye su costo de traslado de la fuente al hogar y su purificación, y es el mismo gobierno el que define el nivel de subsidio de las tarifas de acuerdo con los objetivos que persiga.

La política de distribución del agua en la CDMx ha transitado de entregarla prácticamente gratis a los hogares, a la recuperación de un porcentaje creciente de sus gastos de operación a la fecha. Uno de los objetivos que persigue es la equidad en el suministro del agua a toda la población, principalmente a los hogares pobres. 
En la actualidad, la Ciudad de México es una de las urbes de México con mayores niveles de cobertura en infraestructura de abastecimiento de agua potable (cerca de 98\%). Sin embargo, aún existen alrededor de 350 mil familias que no están conectadas al servicio público, obteniendo el recurso por tandeo con pipas, pozos comunitarios o por acarreo manual (INEGI, 2010; 2015). Las restricciones económicas (en parte, debidas a los subsidios) y la escasez del recurso restringen la expansión y mejoramiento de la oferta en las delegaciones más pobres (Jiménez et al., 2011).

En la CDMX, las viviendas con menor acceso al agua potable son las de mayores niveles de pobreza (gráfica $1 \mathrm{~A}$ ), pero al mismo tiempo, las delegaciones con menores niveles de pobreza se caracterizan por los más altos niveles de igualdad (gráfica 1B). Esto sugiere que existe una relación inversa entre el porcentaje de viviendas con agua entubada y el porcentaje de población en pobreza para las diferentes delegaciones. En consecuencia, los subsidios al consumo de agua potable en la CDMX, cuyo promedio anual asciende a 340 millones de dólares entre los años 2011-2013 (gráfica 2), no se traducen en un beneficio para las personas con menores ingresos económicos o con mayores niveles de necesidades. ${ }^{1}$

Los subsidios siempre han estado presentes en las tarifas de agua en México, y en especial en la CDMX. En 1992 la CDMX enfrentó una severa crisis en el sector del agua debido al deterioro de la infraestructura y las condiciones económicas, sumada a la ineficiencia y al sistema de cobros basados en tarifas fijas, fuertemente subsidiadas por el Estado. Desde entonces, ha surgido la necesidad de eliminar los altos subsidios por razones financieras y con fines de una mejor administración y conservación del recurso (Tortajada, 2006). En 2010 se implementaron tarifas diferenciadas por vez primera y se restructuraron las tarifas para ser más asertivos en el destino de los subsidios. A la fecha las tarifas se aplican según el nivel socioeconómico de los hogares.

El monto total anual del subsidio al agua potable en la CDMX se ha reducido en alrededor de 38 por ciento entre los años 2008 y 2013 (gráfica 2), en vista de la política presupuestal en el país. Para el ejercicio presupuestal de 2017 se está considerado en el Presupuesto de Egresos de la Federación un recorte de 72 por ciento en los servicios de agua potable, al pasar de 12500 a 3400 millones de pesos. En esas condiciones, el Sacmex se verá afectado al reducirse en dicho por-

\footnotetext{
${ }^{1} \mathrm{El}$ subsidio corresponde a la diferencia entre el presupuesto ejercido y los ingresos recaudados por el organismo operador, donde los ingresos recaudados corresponden a la suma por la prestación de servicios por el suministro de agua más los servicios de construcción y operación hidráulica más la autorización para usar las redes de agua y drenaje.
} 
GRÁFICA 1. Acceso a agua potable y pobreza para las diferentes delegaciones de la Ciudad de México
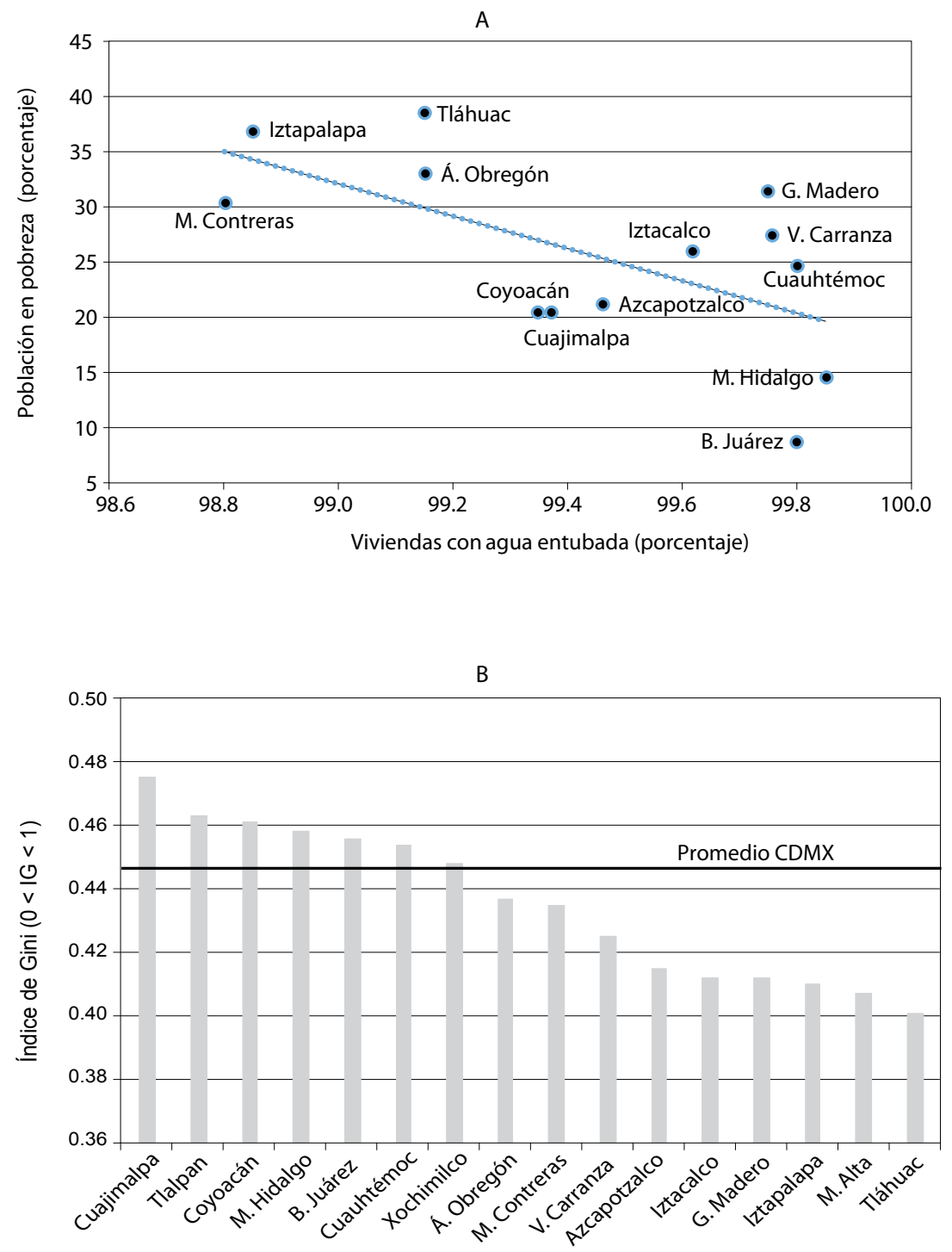

Fuente: Elaboración propia con base en INEGI, 2010 y en la ENIGH, 2010, 2012, 2016. 
GRÁFICA 2. Monto anual del subsidio al agua en la Ciudad de México (millones de dólares)

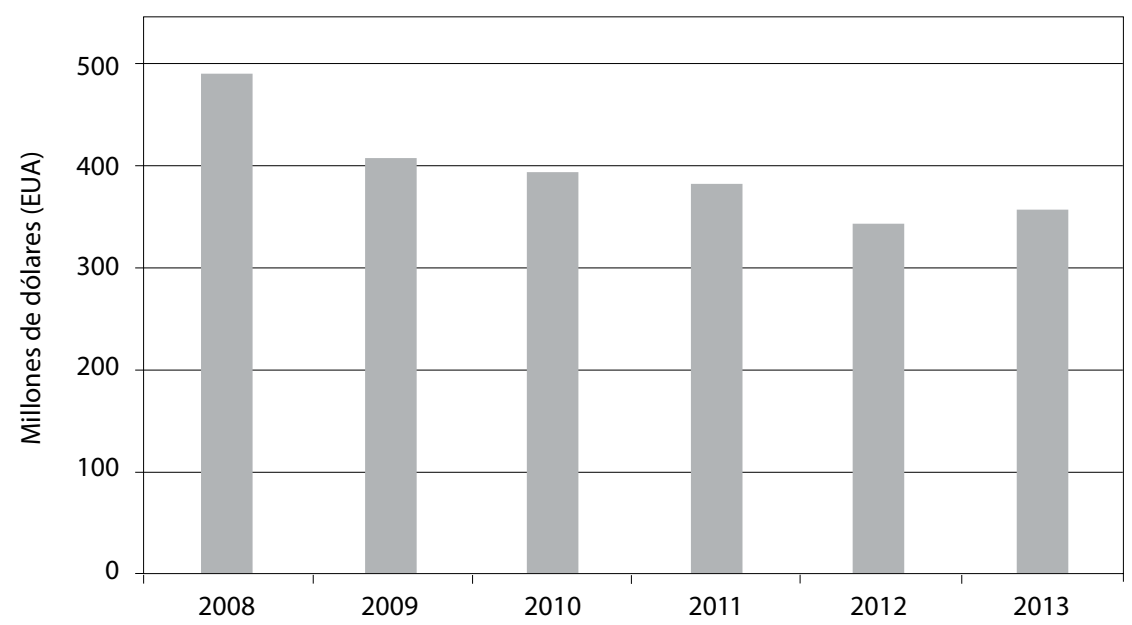

Fuente: Elaboración propia con base en JGDF $(2011,2012)$, GCDMX $(2012,2016)$.

centaje la recepción de subsidios, problema grave, ya que las tarifas actuales son insuficientes para cubrir los costos mínimos de operación del sistema. Además, Sacmex se enfrenta a que cualquier ajuste de tarifas debe ser aprobado por la legislatura local que basa su decisión, en gran parte, en criterios políticos, sin considerar los costos de suministro del servicio y el mantenimiento de la infraestructura hidráulica.

Frente a semejante escenario, este trabajo tiene como objetivo principal examinar si los subsidios destinados al consumo de agua potable de los hogares en la CDMx contribuyen a la reducción de la pobreza, y si su diseńo y aplicación permiten que se distribuyan equitativamente entre los diversos grupos socioeconómicos. El artículo se integra por cinco secciones incluyendo la introducción. En la segunda sección se presenta información sobre el sector de agua potable en la CDMX, seguida por la metodología aplicada, donde se describen las herramientas utilizadas en el artículo. En la cuarta sección se presentan los resultados obtenidos. En la quinta, se ofrecen las conclusiones e implicaciones de la política pública y se recupera alguna experiencia útil para la gestión de los eventuales subsidios al agua de uso doméstico. 


\section{GESTIÓN DEL AGUA POTABLE EN LA CIUDAD DE MÉXICO (CDMX)}

La CDMX es parte de una megaurbe (Zona Metropolitana del Valle de México) que alberga 8851080 habitantes (INEGI, 2010) en un área de $1,495 \mathrm{~km}^{2}$, y genera el 16.5 por ciento de la producción total de México. Sin embargo, a pesar de su importante participación económica, la inequidad en los ingresos en un gran número de hogares y los niveles de pobreza consecuentes se consideran temas críticos (Coneval, 2012). Para atender la demanda de agua potable de los habitantes de la CDMX, se suministra un caudal promedio de $32.3 \mathrm{~m}^{3} /$ segundo, cuya distribución es responsabilidad de un único organismo operador de carácter público, denominado Sistema de Aguas de la Ciudad de México (Sacmex). El agua potable que distribuye Sacmex en 67 por ciento está suministrada por fuentes subterráneas ( $55 \%$ del acuífero del Valle de México y $12 \%$ del Sistema del Lerma, ubicado a $70 \mathrm{~km}$ de distancia). El restante 33 por ciento se obtiene de fuentes superficiales, 3 por ciento de manantiales ubicados en la zona surponiente de la ciudad, y 30 por ciento del sistema Cutzamala ubicado en el Estado de México y Michoacán a $124 \mathrm{~km}$ de distancia (Rodríguez-Tapia et al., 2016).

La distribución de agua entubada a los hogares de la CDMX registra una cobertura de infraestructura de abastecimiento de cerca de 98 por ciento (INEGI, 2010) que es de las más altas del país; sin embargo, la calidad en el servicio que proporciona aún constituye una preocupación fundamental, en particular en lo que tiene que ver con la calidad del agua que se suministra (Espinosa-García et al., 2015). De acuerdo con la Encuesta sobre Hábitos de Consumo, Servicio y Calidad del Agua de los Hogares del Distrito Federal (eHCSCA, 2011), los hogares reportan graves ineficiencias en el servicio recibido, entre las que destaca la elevada discontinuidad del suministro del agua, la cantidad de agua suministrada y la gran desconfianza en la calidad del líquido recibido, además de detectar que se maneja una infraestructura obsoleta y sin el adecuado mantenimiento (Rodríguez-Tapia et al., 2016). Algunos estudios indican que el manejo y el mantenimiento del sistema de distribución es inadecuado e insuficiente, pues se estima que aproximadamente 40 por ciento del agua se pierde en fugas (Morales-Novelo y Rodríguez-Tapia, 2007; Perló y González, 2005) o que presenta variaciones importantes en la calidad (Mazari-Hiriart et al., 2005).

En México las tarifas por el servicio de agua son independientes y establecidas en cada municipio de México (Soto-Montes de Oca, 2015). En el caso de la CDMX, las tarifas se han configurado en una estructura de bloques incrementales, diferenciadas por tipo de usuario final, y emitidas bimestralmente; dicha estructura es la más empleada en el mundo principalmente en países menos desarro- 
Daniel A. Revollo-Fernández, Lilia Rodríguez-Tapia y Jorge A. Morales-Novelo

CUADRO 1. Tarifas para uso doméstico de agua en la CDMX 2010

\begin{tabular}{lcccc}
\hline \multicolumn{2}{c}{ Consumo en litros } & & \multicolumn{2}{c}{ Tarifa (pesos mexicanos) } \\
\cline { 1 - 2 } \cline { 5 - 5 } Limite inferior & Limite superior & & Cuota minima & Cuota adicional \\
\hline 1.0 & 15000 & & 359.08 & 0.00 \\
2. Mayor a 15000 & 20000 & & 359.08 & 23.94 \\
3. Mayor a 20000 & 30000 & & 478.77 & 23.94 \\
4. Mayor a 30000 & 40000 & & 718.15 & 23.94 \\
5. Mayor a 40000 & 50000 & & 957.54 & 23.94 \\
6. Mayor a 50000 & 70000 & & 1196.92 & 29.14 \\
7. Mayor a 70000 & 90000 & & 1779.77 & 31.74 \\
8. Mayor a 90000 & & & 2414.66 & 42.15 \\
\hline
\end{tabular}

Fuente: Elaboración propia con base en ALDF (2011, art. 172). *Cuota adicional por cada 1000 litros excedentes al límite inferior.

llados o emergentes (Barde y Lehmann, 2014; Whittington, et al., 2015). A partir del año 2010, con el objetivo de aumentar la recaudación del sector y reducir el sobreconsumo a consecuencia de precios bajos, la Asamblea Legislativa del Distrito Federal (ALDF) aprobó ajustes al alza en las tarifas que aplica el Sacmex y son cobradas por la tesorería local (Soto-Montes de Oca, 2015). La tarifa para uso doméstico que cobra el Sacmex está constituida por ocho bloques, cada uno con un límite inferior y superior en volumen (litros), y al mismo tiempo cada bloque presenta una cuota mínima y una cuota adicional por cada mil litros excedentes al límite inferior (cuadro 1). Esta estructura tarifaria es progresiva puesto que el cobro aumenta a medida que se genera un mayor consumo de agua en los hogares, con lo cual se trata de reducir el consumo excesivo e indirectamente se intenta que los usuarios valoren mejor dicho recurso.

Por su parte, el Sacmex aplica una política de subsidios al consumo de agua de los hogares que trata de favorecer a los hogares de menores ingresos (cuadro 2). Los subsidios se focalizan de acuerdo con el tipo de vivienda familiar, que se clasifican considerando su correspondiente Índice de Desarrollo por Manzana, que a su vez se determina según el ingreso de los hogares y el valor catastral de las viviendas (Soto-Montes de Oca, 2015). La focalización se basa en la clasificación de las viviendas a partir de la calificación de la manzana en que se ubica; se determinan cuatro grupos de manzanas que definen el porcentaje de subsidio: a) popular, b) baja, $c$ ) media y d) alta. Queda claro, a partir del cuadro 2, que los subsidios aplicados evidencian una relación inversa con el volumen de agua con- 
CUADRO 2. Porcentajes de subsidios que reciben los usuarios a través de las tarifas por tipo de clasificación de la vivienda

\begin{tabular}{|c|c|c|c|c|c|c|c|c|c|}
\hline \multicolumn{2}{|c|}{ Consumo en litros } & \multicolumn{2}{|c|}{ a) Sub. popular (\%) } & \multicolumn{2}{|c|}{ b) Sub. baja (\%) } & \multicolumn{2}{|c|}{ c) Sub. media (\%) } & \multicolumn{2}{|c|}{ d) Sub. alta (\%) } \\
\hline 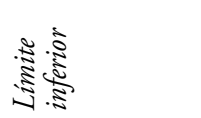 & 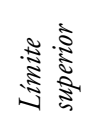 & 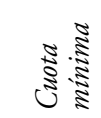 & 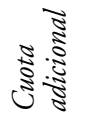 & 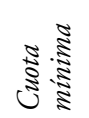 & 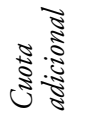 & 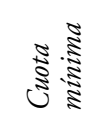 & 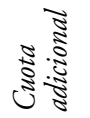 & 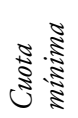 & 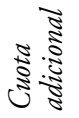 \\
\hline 1. 0 & 15000 & 91.3 & 0.0 & 90.1 & 0.0 & 67.4 & 0.0 & 60.9 & 0.0 \\
\hline 2. Mayor a 15000 & 20000 & 91.3 & 88.7 & 90.1 & 74.8 & 67.4 & 36.9 & 60.9 & 33.9 \\
\hline 3. Mayor a 20000 & 30000 & 90.6 & 82.7 & 86.3 & 69.6 & 59.8 & 36.9 & 54.1 & 33.8 \\
\hline 4. Mayor a 30000 & 40000 & 87.9 & 66.1 & 80.7 & 69.5 & 52.1 & 36.8 & 47.4 & 33.8 \\
\hline 5. Mayor a 40000 & 50000 & 82.5 & 66.1 & 77.9 & 38.3 & 48.3 & 36.8 & 43.9 & 33.7 \\
\hline 6. Mayor a 50000 & 70000 & 79.2 & 25.3 & 70.0 & 25.0 & 46.0 & 18.8 & 41.9 & 17.1 \\
\hline 7. Mayor a 70000 & 90000 & 61.6 & 24.6 & 55.3 & 24.6 & 37.1 & 1.6 & 33.8 & 1.6 \\
\hline 8. Mayor a 90000 & & 51.8 & 1.2 & 47.2 & 1.2 & 27.8 & 1.2 & 25.3 & 1.2 \\
\hline
\end{tabular}

Fuente: Elaboración propia con base en ALDF (2011, art. 172).

sumida y una relación directa con el nivel de pobreza de los hogares. Sin embargo, interesa destacar el hecho que todos los hogares, con independencia de su nivel de ingreso, se benefician de algún modo con los subsidios.

\section{Metodología}

La metodología aplicada consiste en la estimación de cuatro indicadores que miden la incidencia de los subsidios al agua en los hogares de la CDMX; el primer indicador es el $a$ ) análisis de incidencia de subsidio (AIS) que capta su impacto sobre la reducción de la pobreza; seguido de un grupo de tres indicadores que engloban el análisis de inequidad en la distribución (AID) que muestran cómo esta política pública impacta a diversos grupos socioeconómicos, los indicadores considerados en este grupo son: $b$ ) indicador de focalización $(\Omega), c$ ) indicador de concentración (IC) y d) curva de concentración (CC). Se recurre a estos cuatro indicadores como recomienda la literatura internacional (Nina, 2011).

\section{Análisis de incidencia de subsidio (AIS)}

El análisis de incidencia de subsidio (AIs) mide la distribución de este gasto gubernamental entre los distintos grupos de hogares con diferentes niveles sociales (Cuenca, 2008). Para estimar el AIS se siguen tres momentos: a) identifi- 
cación de los usuarios del agua de uso doméstico y el monto pagado por el servicio, b) clasificación de los hogares en grupos socioeconómicos - el criterio es el ingreso familiar total como proxy de medida de bienestar- y c) con dicha información se estima la ecuación del indicador expresada en la ecuación 1 (Demery, 2000; Cuenca, 2008).

$$
A I S_{j}=X_{j}=\sum_{i=1}^{m}\left\{\frac{\left(E_{i j} / E_{i}\right) * S_{i}}{\sum_{j=1}^{n}\left[\left(E_{i j} / E_{i}\right) * S_{i}\right]}\right\}=\sum_{i=1}^{m}\left[\frac{e_{i j}{ }^{*} S_{i}}{\sum_{j=1}^{n}\left(e_{i j}{ }^{*} S_{i}\right)}\right]
$$

El indicador AIs $\left(X_{j}\right)$ es el porcentaje del subsidio al agua del grupo beneficiado " $j$ ", $S$ es la cantidad del subsidio otorgado por el gobierno, $E$ es el gasto de los hogares en bienes y servicios, e " $i$ " es el bien o servicio en cuestión (en este caso el bien es el agua potable, por ende " $i$ " $=1$ ). Por lo tanto, $e_{i j}$ representa la proporción del gasto del hogar " $j$ " en el bien " $i$ " del total del gasto de los hogares en el bien " $i$ "; mientras que $S_{i}$ representa el subsidio total del gobierno en el bien o servicio “"

\section{Análisis de inequidad en la distribución (AID)}

Para determinar la inequidad en la distribución del subsidio entre la población se estiman tres indicadores, el indicador de focalización $(\Omega)$, el indicador de concentración (IC) y la curva de concentración (CC) (Vélez, 1996; Nina, 2011).

Indicador de focalización $(\Omega)$. El indicador de focalización se expresa en la ecuación 2 y mide la proporción de los subsidios que recibe un grupo de hogares, dividida entre la proporción de subsidios de la población total.

$$
\Omega=\frac{S_{p} / P}{S_{H} / H}=\left(\frac{S_{p}}{S_{H}}\right)\left(\frac{H}{P}\right)
$$

Donde $S_{P}$ es el valor de los subsidios recibidos por los hogares pobres, $S_{H}$ es el valor total del subsidio que reciben en conjunto los hogares, $P$ es el número de hogares pobres y $H$ es el número total de hogares. Un valor igual a uno de $\Omega$ implica que la distribución del subsidio es neutral, siendo la proporción de los beneficios que llegan a los hogares pobres igual a la proporción de la población que conforma esos hogares. Un valor mayor de uno implica que la distribución del subsidio es progresiva; es decir, los hogares pobres se benefician de una proporción de los beneficios totales mayor que la proporción que representan en la 
población. Finalmente, si $\Omega$ es inferior a uno implica que la distribución del subsidio es regresiva.

La curva de concentración (CC) y el indicador de concentración (IC). Para medir la desigualdad en la distribución de los subsidios entre los hogares, la literatura sugiere estimar el indicador de concentración (IC) asociado a la curva de concentración (cc) (Vélez, 1996; Nina, 2011). El IC es similar al coeficiente de Gini asociado a la curva de Lorenz, construida a partir de la distribución del ingreso del hogar. ${ }^{2}$ En este caso, la cc corresponde a la función de distribución acumulada de los subsidios manteniendo el ordenamiento inicial de los hogares de manera ascendente por su ingreso (gráfica 3). Mientras que el IC se define como el área entre la curva de concentración y la curva de concentración igualitaria (CCI).

GRÁFICA 3. Curva de concentración (CC) e indicador de concentración (IC)

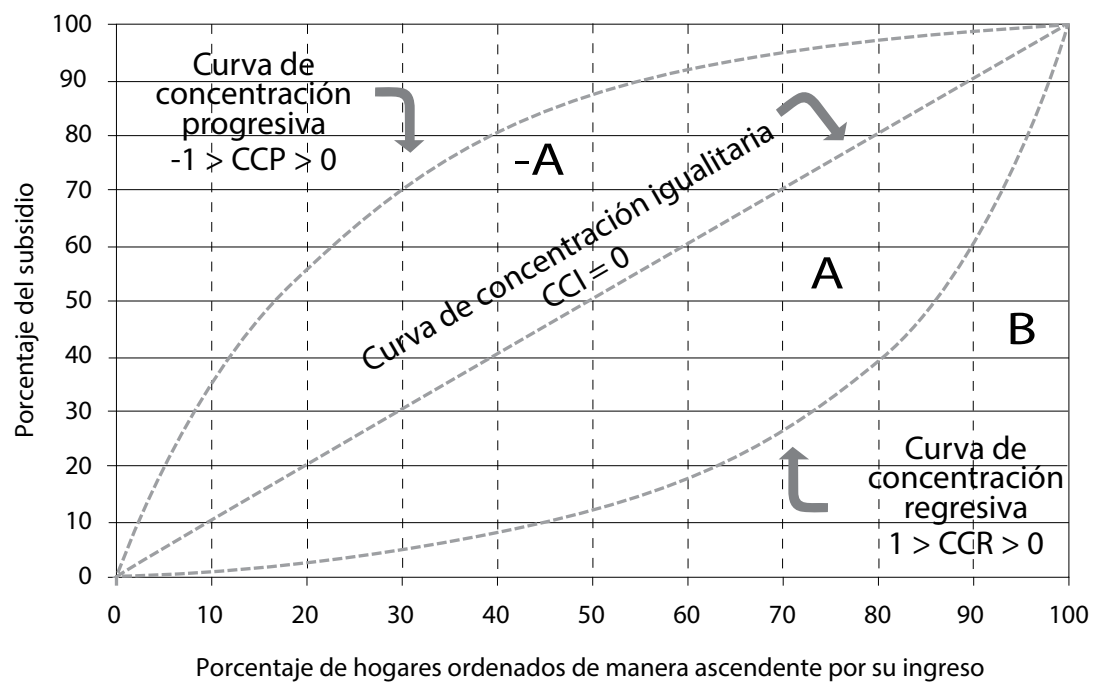

Fuente: Elaboración propia, 2016.

${ }^{2}$ La curva de Lorenz relaciona la proporción acumulada de ingresos con la proporción acumulada de población. Esta curva es una representación gráfica de la distribución del ingreso y se compara con una recta de $45^{\circ}$ que une el origen $(0,0)$ con el punto $(100,100)$, denominada curva de perfecta igualdad, que refleja una relación igual entre la proporción de individuos y de ingreso acumulado. Cuanto más alejada esté la curva de Lorenz con respecto a la curva de perfecta igualdad, más desigual resultará la distribución del ingreso. 
Cabe destacar que, a diferencia de la curva de Lorenz, la cC puede ubicarse por encima de la CCI, por lo cual el indicador de concentración varía entre -1 y +1 . En este caso, número negativos indican una distribución favorable a los pobres (pro-pobre); mientras que valores positivos indican una distribución que favorece a los hogares de mayores ingresos (pro-rica) (Gasparini et al., 2012). De esta forma, el indicador de concentración se puede calcular a partir de la ecuación 3.

$$
I C=\frac{A}{A+B} \quad ;-1<I C<+1
$$

CUADRO 3. Resumen de indicadores empleados para medir el impacto del subsidio sobre los hogares de CDMX

\begin{tabular}{|c|c|c|}
\hline $\begin{array}{l}\text { Indicador } \\
\text { Grupo }\end{array}$ & Definición & Medición \\
\hline a) Análisis de incidencia de subsidio (AIS) & $\begin{array}{l}\text { Mide la distribución de este gasto } \\
\text { entre los distintos grupos de hogares } \\
\text { con diferentes niveles sociales. }\end{array}$ & Porcentual \\
\hline \multicolumn{3}{|l|}{ b) Análisis de inequidad en la distribución (AID) } \\
\hline 1. Indicador de focalización $(\Omega)$ & $\begin{array}{l}\text { Proporción de subsidios que recibe } \\
\text { un grupo de hogares, dividida entre } \\
\text { la proporción de subsidios de la } \\
\text { población total. }\end{array}$ & $\begin{array}{l}\Omega=1 \text { : Distribución normal; } \\
\Omega>1 \text { : Distribución progresiva; } \\
\Omega<1 \text { : Distribución regresiva. }\end{array}$ \\
\hline 2. Indicador de concentración (IC) & \multirow{2}{*}{$\begin{array}{l}\text { Permite medir la desigualdad en la } \\
\text { distribución de los subsidios entre } \\
\text { los hogares. }\end{array}$} & \multirow{2}{*}{$\begin{array}{l}\text { Número negativo indica una } \\
\text { distribución favorable a los } \\
\text { pobres, valor positivo indica } \\
\text { una distribución que favorece } \\
\text { a los hogares de mayores } \\
\text { ingresos: }-1<c c<+1 \text {. }\end{array}$} \\
\hline 3. Curva de concentración (cc) & & \\
\hline
\end{tabular}

Fuente: Elaboración propia, 2016.

\section{Datos}

Los datos utilizados en esta investigación se obtuvieron básicamente de la Encuesta de Hábitos de Consumo, Servicio y Calidad del Agua de los Hogares del Distrito Federal, 2011 (eHCSCA), además del Censo de Población y Vivienda (INEGI, 2010) y de la Encuesta Nacional de Ingresos y Gastos de los Hogares (ENIGH, 2010, 2012, 2014). Se recurre básicamente a la EHCSCA debido a que reporta información especializada y exclusiva sobre consumo de agua para los hogares de la CDMX, básicamente se aplicó a familias que residen permanentemente en viviendas particulares de la CDMX entre agosto y septiembre de 2011. 
La EHCSCA se basa en una muestra de 689 viviendas elegidas aleatoriamente con un nivel de confianza de 99 por ciento y con un margen de error de 5 por ciento, representativa de un marco poblacional de 1903983 viviendas que contaban con agua entubada.

Las estimaciones de los indicadores y curvas de concentración se realizaron en tres tipos de agrupación de los hogares. En primer lugar, se clasificaron los hogares de acuerdo con sus ingresos económicos bimensuales en bajo (menores a 3000 pesos), medio bajo (3001 a 6500 pesos), medio alto (6501 a 9000 pesos) y alto (9001 o más); la segunda clasificación de los hogares fue en función de las tarifas de agua que pagan bimensualmente (popular, baja, media y alta) y finalmente en una clasificación que combina los ingresos y las tarifas. Es importante indicar que para la clasificación de los ingresos económicos en esos cuatro grupos se consideraron dos aspectos: a) se trató de dividir la muestra de los datos en cuatro cuantiles y $b$ ) se tomaron en cuenta otras clasificaciones de otras organizaciones, como por ejemplo la que realiza el Banco Mundial. El Banco Mundial clasifica a los países en cuatro grupos según el producto interno bruto (PIB per cápita al año): a) economías de bajos ingresos (1 005 EUA o menos), b) economía de ingresos medio-bajo (1 006-3955 EUA), c) economía de ingresos medio-alto (395612235 EUA) y d) economía de altos ingresos (12236 EUA o más). México está clasificado en el grupo de economía de ingresos medio-alto.

\section{RESULTADOS Y DISCUSIÓN}

\section{Análisis de incidencia del subsidio (AIS) al agua potable}

De acuerdo con la encuesta aplicada a hogares en la CDMX en 2011 (EHCSCA) y a su clasificación (cuadro 4), más de la mitad de los 1.9 millones de hogares se encuentran en el rango de ingresos medios (aproximadamente 56 por ciento expresan que disponen aproximadamente entre 3001 y 9000 pesos al mes como ingreso económico). Los hogares pobres (ingresos menores a los 3000 mensuales) explican una tercera parte del total de hogares $(30.7 \%)$ y los hogares de ingresos altos (ingresos mayores a 9000 pesos mensuales) explican arriba de una décima del total (13.5\%). El indicador AIs que mide la participación ponderada del subsidio al agua potable de los hogares (ponderada por su correspondiente gasto en agua) (columna 3, cuadro 4), indica que el grupo de ingresos medio bajo capta casi 43 por ciento del subsidio, seguido por el grupo de ingresos medio alto con 26.5 por ciento, el de ingresos altos capta una quinta parte del total; contrastando con la baja participación (10.5\%) del grupo de hogares de ingresos bajos, y que muestra que este grupo es el que registra la menor incidencia en su 
CUADRO 4. Análisis de incidencia de subsidios al agua potable en los hogares de la Ciudad de México, 2011

\begin{tabular}{lccc}
\hline $\begin{array}{c}\text { Ingresos } \\
\text { (1) Rango ingreso } \\
\text { familiar }\end{array}$ & $\begin{array}{c}\text { (2) Distribución } \\
\text { de hogares }(\%)\end{array}$ & $\begin{array}{c}\text { (3) Incidencia } \\
\text { de subsidio (\%) }\end{array}$ \\
\hline Bajo & Menores a 3000 & 30.70 & 10.50 \\
Medio bajo & 3001 a 6500 & 36.50 & 42.90 \\
Medio alto & 6501 a 9000 & 19.30 & 26.50 \\
Alto & 9001 o más & 13.50 & 20.10 \\
Total & & 100.00 & 100.00 \\
\hline
\end{tabular}

Fuente: Elaboración propia con base en EHCSCA, 2011. ${ }^{*}$ Donde $i$ es el servicio, en este caso consumo de agua potable, y $j$ es el grupo beneficiado ( $j=$ bajo, medio bajo, medio alto, alto).

bienestar ante el otorgamiento de los subsidios. Como se muestra más adelante, este resultado se explica porque los hogares con menores ingresos económicos registran los más bajos niveles de acceso al servicio de agua potable y a que el diseño de subsidios no incida sobre estos hogares de forma importante.

\section{Análisis de inequidad en la distribución (AID)}

Indicador de focalización $(\Omega)$. El indicador de focalización $(\Omega)$ para los hogares con ingreso bajo es menor a uno (0.92) (cuadro 5, renglón 1), que indica una distribución regresiva del subsidio en este grupo; para el caso de un conjunto de hogares más amplio en que se agrupan los hogares con ingresos bajos y medio bajos el indicador $\Omega$ continúa siendo menor a uno (0.97), manteniendo su carácter regresivo. Para los dos subgrupos de hogares mencionados el valor de $\Omega$ se encuentra debajo de uno; mostrando que los hogares de menores ingresos en la

CUADRO 5. Indicador de focalización del subsidio al agua potable $(\Omega)$ para Ciudad de México

\begin{tabular}{lccc}
\hline \multicolumn{1}{c}{ Hogares } & Ingreso & $\Omega^{*}$ & Distribución del subsidio \\
\hline Bajo & Menores a 3000 & 0.90 & Regresiva \\
Medio bajo & 3001 a 6500 & 1.00 & Neutral \\
Medio alto & 65001 a 9000 & 1.00 & Neutral \\
Alto & 9001 o más & 1.10 & Progresiva \\
Bajos + Medio bajo & Menor a 6500 & 0.97 & Regresiva \\
\hline
\end{tabular}

Fuente: Elaboración propia con base en la EHCSCA, 2011 . $^{*} \Omega=1$ distribución del subsidio es neutral, $\Omega>1$ distribución del subsidio es progresiva, $\Omega<1$ distribución del subsidio es regresiva. 
CDMX se benefician de una menor proporción de los subsidios respecto a la proporción de población que representan (distribución regresiva).

Para los grupos de ingresos más altos, el indicador de focalización aumenta a medida que el ingreso familiar crece. Para los grupos de ingreso medio bajo y medio alto el valor de su $\Omega$ es igual a uno, reflejando una distribución neutral; la situación mejora en el caso de los hogares del grupo de ingreso alto en los que su $\Omega$ es igual a 1.1, lo que indica una distribución progresiva (cuadro 5).

Indicador de concentración (IC). Este indicador se estimó a partir de información de la EHCSCA para dos variables, los subsidios y el agua suministrada a los hogares en metros cúbicos. Por lo tanto cada grupo de hogares registra tanto el indicador de concentración (IC) para los subsidios como respecto al agua suministrada (metros cúbicos). Los IC se estimaron para dos niveles, primero a nivel de toda la población de la CDMx y después para cada uno de los grupos de hogares siguiendo la clasificación de hogares que utiliza el Sistema de Aguas de la Ciudad de México (SACM) en la aplicación del sistema tarifario doméstico (popular, baja, media y alta) (cuadro 6). Como se señaló arriba, los IC varían entre -1 y +1 , los números negativos indican una distribución pro-pobre y los positivos una distribución pro-rico.

Los IC estimados para toda la población son positivos (cuadro 6), lo que evidencia que tanto el volumen de agua suministrada $(\mathrm{IC}=0.437)$ como los subsidios otorgados al consumo de agua doméstica $(\mathrm{IC}=0.429)$ se distribuyen a favor de los grupos de hogares de mayores ingresos en la CDMX (distribución pro-rica). Los IC de ambas variables registran valores muy similares, pero es ligeramente mayor la concentración de los volúmenes de agua, lo que muestra que los subsidios se distribuyen directamente a partir del acceso al agua entubada, lo que no

CUADRO 6. Coeficiente de concentración (IC) estimado para Ciudad de México: metros cúbicos consumidos y subsidio recibido

\begin{tabular}{lcc}
\hline & IC $m^{3 *}$ & IC Subsidio $^{*}$ \\
\hline Popular & 0.48 & 0.464 \\
Baja & 0.418 & 0.417 \\
Media & 0.439 & 0.428 \\
Alta & 0.336 & 0.328 \\
Población total CDMx & 0.437 & 0.429 \\
\hline
\end{tabular}

Fuente: Elaboración propia con base en la EHCSCA, 2011. ${ }^{*}-1<$ IC $<+1$, pro-pobre $<$ IC $<$ pro-rica. 
corresponde al diseño de las tarifas que se supone pretende beneficiar a los hogares de menores ingresos.

$\mathrm{Al}$ analizar los IC al interior de cada grupo de hogares, se evidencia que los hogares que se clasifican en tarifa popular presentan un IC mayor que el resto de los grupos; tanto en el consumo de agua en $\mathrm{m}^{3}(\mathrm{IC}=0.480)$ como en el subsidio $(\mathrm{IC}=0.464)$, lo que implica que este grupo de hogares registra una gran heterogeneidad en su interior respecto a las variables en estudio, que se explica por una interesante disparidad en los niveles de ingresos económicos entre hogares y que por lo tanto en este grupo existen hogares que se ven beneficiadas por mayores niveles de subsidios que los que estrictamente les corresponden. Los grupos de hogares bajo y medio se benefician, ya que sus IC son de 0.417 y 0.428 respectivamente. Destaca que el grupo de hogares clasificado en tarifa alta registre la mayor homogeneidad en la distribución del subsidio ( $\mathrm{IC}=0.328$ ) y del volumen de agua consumida $(\mathrm{IC}=0.336)$, lo que sugiere un menor problema de clasificación en este estrato.

Los IC estimados confirman que la actual estructura tarifaria del agua y su implementación favorece en mayor medida a los hogares de ingresos medios y altos en el otorgamiento de subsidios. Lo que posiblemente se explica porque los subsidios otorgados en las tarifas popular, baja y media se aplican erróneamente a hogares que registran ingresos mayores que los que justifican su aplicación.

\section{Curvas de concentración (CC) del consumo y de los subsidios al agua para los hogares de la CDMX}

La curva de concentración tanto del agua consumida $\left(\mathrm{m}^{3}\right)$ como de los subsidios (gráfica 4) se construye para grupos de hogares (veintiles) de acuerdo con su nivel del ingreso familiar registrado en la EHCSCA (eje de las X). Se evidencia que el primer veintil de hogares de menores ingresos económicos consumen aproximadamente 7 por ciento del total de agua suministrada en la CDMX (metros cúbicos) y reciben cerca de 8 por ciento del total de los subsidios. En el otro extremo, el quinto veintil ( $20 \%$ de la población de mayores ingresos) consume casi 42 por ciento del agua suministrada en la CDMx y 40 por ciento del total de los subsidios (gráfica 4). Esta distribución muestra que tanto el consumo de agua como la recepción de los subsidios favorece en mayor medida a los hogares de ingresos más altos. La inequidad en la distribución del agua a los hogares pobres se reafirma al contrastar el consumo per cápita bimestral de $10.5 \mathrm{~m}^{3}$ en un hogar pobre en tanto que una persona de un hogar con ingresos altos consume aproximadamente $12.2 \mathrm{~m}^{3}$ (gráfica 5). Este comportamiento confirma la alta correlación que existe entre el consumo de agua en volumen y en los subsidios. 
GRÁFICA 4. Curva de concentración (cC) para la Ciudad de México: subsidio recibido y metros cúbicos consumidos

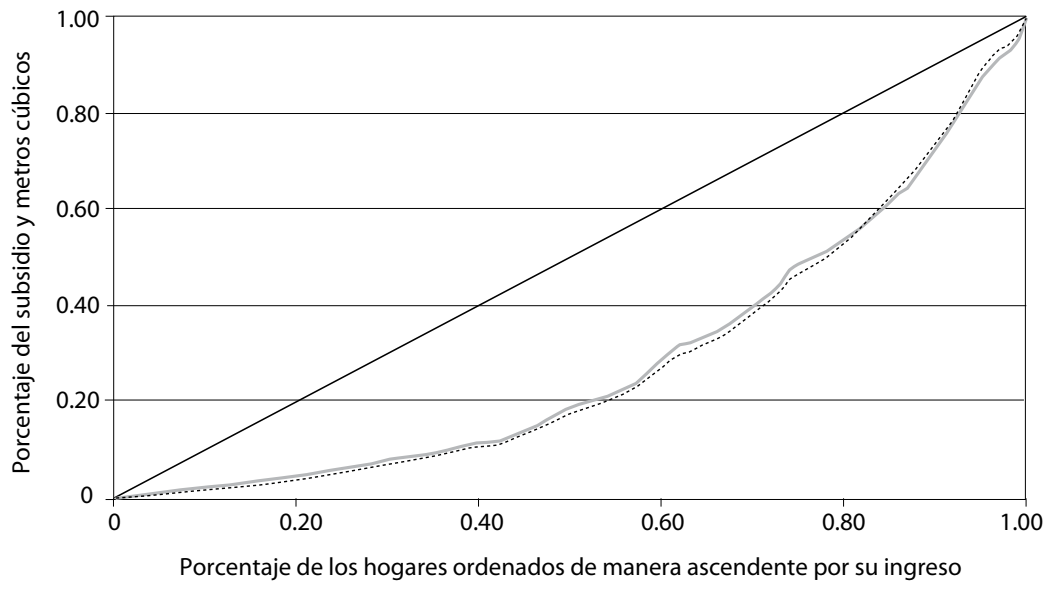

Línea a $45^{\circ}$

Subsidio

$M^{3}$

Fuente: Elaboración propia con base en la EHCSCA, 2011.

GRÁFICA 5. Consumo de metros cúbicos per cápita al bimestre para diferentes grupos económicos

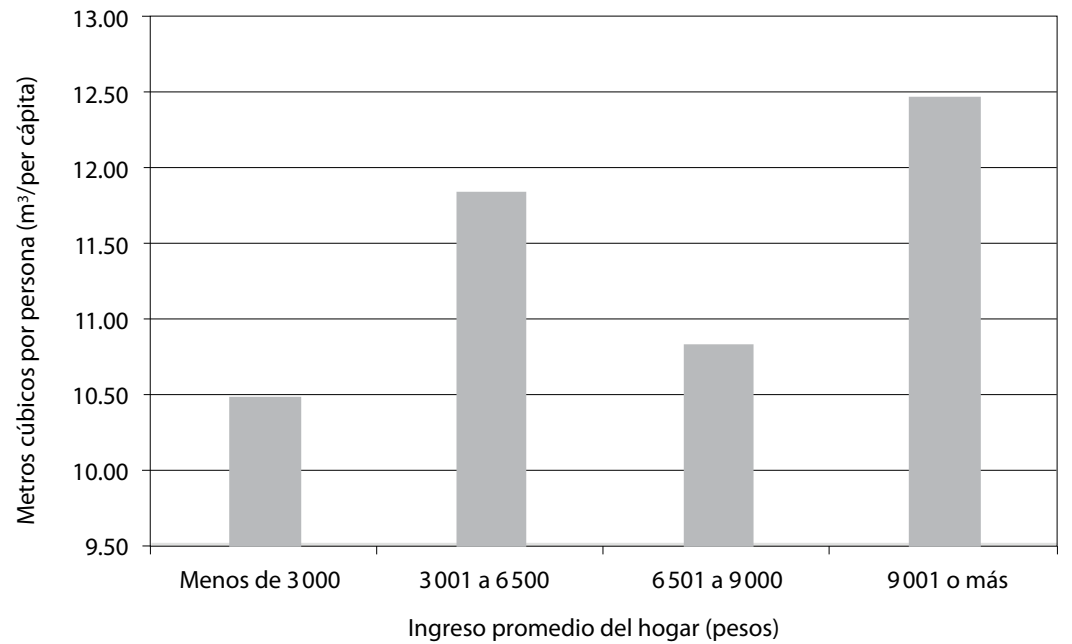

Fuente: Elaboración propia con base en la EHCSCA, 2011. 


\section{Curva de concentración (CC) de hogares con la misma tarifa}

La gráfica 6 presenta cuatro gráficas de curvas de concentración (cc) correspondientes a hogares que acceden a la misma tarifa de agua en la CDMX (popular, baja, media y alta). Las cc de los grupos de hogares clasificados en tarifa popular, baja y media (gráfica 6a, 6b y 6c) evidencian una distribución desigual en los volúmenes de agua consumidos y en los subsidios recibidos, siendo más agudo el caso de la tarifa popular. En este último grupo de hogares (6a), 20 por ciento más pobre se beneficia de aproximadamente 3 por ciento del total de los subsidios (y recibe un porcentaje del agua distribuida con un valor similar); no obstante que sea el grupo de hogares a quien la política hídrica dirige los subsidios para atenuar el problema de la pobreza urbana; en contrapunto, 20 por ciento de los hogares con ingresos más altos se favorece con 56 por ciento de los subsidios y del consumo de agua. Este resultado sugiere que existe un problema en la operación de los subsidios en la tarifa popular, en el sentido de que parte de las mayores tasas de subsidios se canaliza a hogares de ingresos altos, al mismo tiempo que los hogares que lo requieren, de manera prioritaria para mejorar sus condiciones de vida, no lo reciben.

La gráfica $6 \mathrm{~d}$ muestra las cc de los hogares clasificados con tarifa alta y registran mayor equidad tanto en los $\mathrm{m}^{3}$ de agua potable consumida como en los subsidios. Esto es, del total de hogares en este grupo, 60 por ciento de las familias de ingresos medios recibe 59 por ciento del total de subsidios para el grupo y del agua suministrada, mientras que 20 por ciento de los hogares con mayores ingresos económicos se favorecen con 36 por ciento de ambos rubros, aunque 20 por ciento de los hogares de menores ingresos resultan favorecidos con cerca de 5 por ciento del total de los subsidios otorgados al grupo.

Los resultados previos muestran que los subsidios se distribuyen en forma diferenciada entre hogares que pagan la misma tarifa, inclinada a favorecer en forma importante a los hogares de ingresos altos, seguido de los ingresos medios, y en detrimento de los hogares de menores ingresos en la CDMx. Esta inequidad puede deberse a fallas en la adjudicación de la tarifa popular a hogares que pueden pagar una tarifa más alta. Este problema se confirma revisando la clasificación de los hogares y su tarifa de agua potable asignada, ya que de acuerdo con información recolectada por medio de la EHCSCA 2011, existen hogares con altos ingresos económicos que reportan recibir una tarifa popular, y hogares con ingresos económicos bajos que reciben una tarifa alta (cuadro 7). De acuerdo con el cuadro 7, aproximadamente 12.7 por ciento de los hogares clasificados con tarifa popular presentan un ingreso promedio del hogar por encima de los 9000 pesos, que los 
GRÁFICA 6. Curva de concentración (CC) de hogares con la misma tarifa: metros cúbicos consumidos y subsidio al agua en la Ciudad de México

a) Tarifa popular

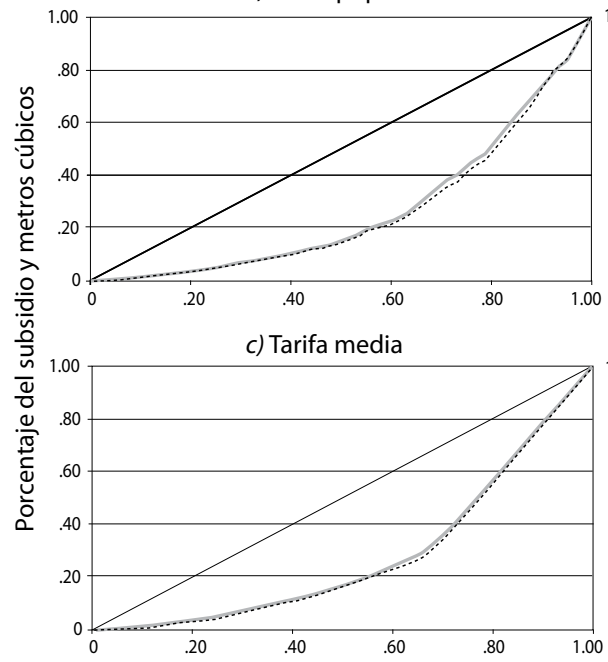

b) Tarifa baja

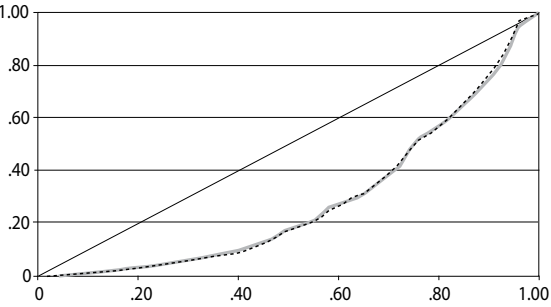

d) Tarifa alta

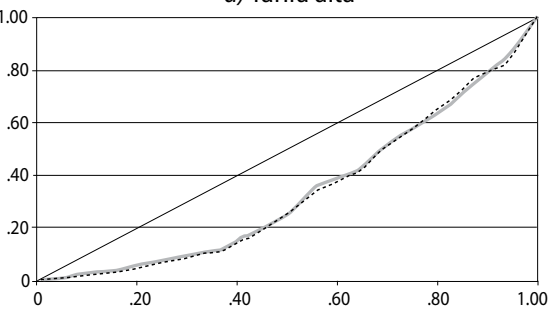

Porcentaje de los hogares ordenados de manera ascendente por su ingreso Línea de $45^{\circ}$ Subsidio $\mathrm{M}^{3}$

Fuente: Elaboración propia con base en EHCSCA, 2011.

CUADRO 7. Clasificación de los hogares usuarios del servicio de agua potable en función del nivel de ingresos económicos y del tipo de tarifa que reciben

\begin{tabular}{|c|c|c|c|c|c|c|}
\hline & & \multicolumn{5}{|c|}{ Por tipo de ingreso (\%) } \\
\hline & & Menores a 3000 & 3001 a 6500 & 6501 a 9000 & 9001 o más & Total \\
\hline & Hogares & Bajo & Medio bajo & Medio alto & Alto & \\
\hline \multirow{4}{*}{ 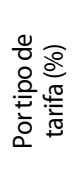 } & Popular & 28.50 & 38.90 & 19.90 & 12.70 & 100.00 \\
\hline & Baja & 28.10 & 37.50 & 23.90 & 10.50 & 100.00 \\
\hline & Media & 3.90 & 53.20 & 30.20 & 12.70 & 100.00 \\
\hline & Alta & 13.10 & 36.20 & 20.60 & 30.10 & 100.00 \\
\hline
\end{tabular}

Fuente: Elaboración propia con base en la EHCSCA, 2011. 
ubica como de ingresos altos. De igual forma, 10.5 por ciento de los hogares clasificados con tarifa baja presentan ingresos altos. Por otro lado, 3.8 por ciento de los hogares clasificados con tarifa media presentan un ingreso menor a 3000 pesos, que los califica como pobres. Y finalmente, 13.1 por ciento de los hogares con tarifa alta cuentan con ingresos bajos (hasta 3000 pesos) que los califica como pobres. En este sentido, no obstante, la focalización ya implementada por el Sacmex es un gran avance, pero es imperativo revisar su diseño e implementación para tratar de evitar esta desviación de los subsidios a hogares que no lo necesitan o que los necesitan en menor medida que los hogares con mayores necesidades.

Los resultados para la CDMX coinciden con los de otros estudios que muestran que el otorgamiento de subsidios al consumo de agua entubada resultan ser poco equitativos al comparar su distribución entre los diferentes grupos de la sociedad. Por ejemplo, Kochhar et al. (2015) estiman que en los países con bajos ingresos, 20 por ciento de los hogares más pobres reciben aproximadamente 12 por ciento del total de los subsidios; mientras que 20 por ciento más rico, recibe aproximadamente 30 por ciento.

\section{CONCLUSIONES}

Los cuatro indicadores estimados para evaluar la incidencia de los subsidios al agua en los hogares de la CDMX evidencian que los hogares pobres han sido los menos beneficiados (cuadro 8). El análisis de incidencia de subsidios (AIs) manifiesta que los hogares clasificados como pobres participan con 10.5 por ciento del total de subsidios de la ciudad. El indicador de focalización $(\Omega)$ para los hogares pobres y el grupo que incluye a estos últimos y a los hogares de ingresos medio bajos es menor a uno (0.97), lo que significa que los hogares de menores ingresos en la CDMX se benefician de una menor proporción de los subsidios respecto a la proporción de población que representan (distribución regresiva).

El indicador de concentración (IC) mide la desigualdad entre grupos de hogares y valores positivos del mismo señalan una situación favorable a los hogares de mayores ingresos. Los coeficientes del estudio son positivos, el IC estimado para el volumen de agua suministrada a los hogares es 0.437 y el IC para los subsidios al agua es de 0.42 , que indican que los grupos de hogares de mayores ingresos en la CDMX se ven más favorecidos en la distribución de ambas magnitudes (distribución pro-rica). El valor tan similar de ambos IC sugiere que los subsidios a los hogares se distribuyen con base en el criterio del volumen de agua consumida, lo que se opone al diseńo de la estructura de los subsidios. 
CUADRO 8. Resumen de los resultados de los indicadores empleados para medir el impacto del subsibio sobre los hogares de CDMX

\begin{tabular}{|c|c|c|c|}
\hline Indicador & Definición & \multicolumn{2}{|c|}{ Medición } \\
\hline \multirow[t]{4}{*}{ Análisis de incidencia de subsidio (AIS) } & $\begin{array}{l}\text { Mide la distribución de este } \\
\text { gasto entre los distintos grupos } \\
\text { de hogares con diferentes } \\
\text { niveles sociales. }\end{array}$ & Ingreso bajo & $10.50 \%$ \\
\hline & & Ingreso medio bajo & $42.90 \%$ \\
\hline & & Ingreso medio alto & $26.50 \%$ \\
\hline & & Ingreso alto & $20.10 \%$ \\
\hline \multicolumn{4}{|c|}{ Análisis de inequidad en la distribución (AID) } \\
\hline \multirow[t]{4}{*}{ Indicador de focalización $(\Omega)$} & \multirow{4}{*}{$\begin{array}{l}\text { Proporción de subsidios que } \\
\text { recibe un grupo de hogares, } \\
\text { dividida entre la proporción de } \\
\text { subsidios de la población total. }\end{array}$} & Ingreso bajo & 0.9 /Regresiva \\
\hline & & Ingreso medio bajo & $1.0 /$ Neutral \\
\hline & & Ingreso medio alto & 1.0/ Neutral \\
\hline & & Ingreso alto & 1.1 / Regresiva \\
\hline \multirow[t]{5}{*}{ Indicador de concentración (IC) } & \multirow{5}{*}{$\begin{array}{l}\text { Permite medir la desigualdad } \\
\text { en la distribución de los } \\
\text { subsidios entre los hogares. }\end{array}$} & Ingreso bajo & 0.464 \\
\hline & & Ingreso medio bajo & 0.417 \\
\hline & & Ingreso medio alto & 0.428 \\
\hline & & Ingreso alto & 0.328 \\
\hline & & Población Total CDMX & 0.429 \\
\hline
\end{tabular}

Fuente: Elaboración propia, 2016.

Los valores de los IC al interior de cada grupo de hogares clasificados por tarifa muestra que los hogares que pagan la tarifa popular presentan un IC mayor que el resto de los grupos; tanto en el consumo de agua en $\mathrm{m}^{3}(\mathrm{IC}=0.480)$ como en el subsidio (IC $=0.464$ ), lo que implica que existe una gran heterogeneidad entre los hogares respecto de ambas variables, que se debe a la gran disparidad en los niveles de ingresos económicos, incluyendo hogares de altos ingresos que no deberían acceder a esta tarifa. Los resultados previos se refuerzan al analizar la curva de concentración (CC) de los hogares que enfrentan la misma tarifa. En esta curva se observa que 12.7 por ciento de los hogares clasificados con tarifa popular presentan un ingreso alto. De igual forma, 10.5 por ciento de los hogares clasificados con tarifa baja presentan ingresos altos; 3.8 por ciento de los hogares clasificados con tarifa media presentan un ingreso que los califica como pobres y finalmente, 13.1 por ciento de los hogares con tarifa alta registran ingresos que los califica como pobres. Lo anterior muestra que existe un error sistemático en 
la aplicación de los subsidios a los hogares, lo que tiene un impacto adverso sobre los hogares pobres y favorable para los hogares de ingresos altos.

Los resultados econométricos de la investigación indican con precisión que los subsidios al agua no están llegando a los hogares más pobres de la ciudad e indica la premura e importancia de que Sacmex redefina su politica de tarifas y cobertura del servicio. Uno de los hallazgos más importantes de la investigación es que con la actual estructura de tarifas los subsidios al agua se distribuyen en relación directa al volumen de agua consumida, y el agua no llega a una parte muy importante de hogares con ingresos más bajos, el estudio precisa las zonas con los problemas más graves de la falta de cobertura de los servicios. Es importante que Sacmex ajuste su politica distributiva e incremente la cobertuta del servicio en áreas pobres y aumente su eficiencia en el servicio y autosuficiencia económica en toda la ciudad.

En este sentido, es imperativo revisar la política de subsidios y su implementación para frenar la desviación de los subsidios a hogares que no lo necesitan y dirigirlo a los hogares con mayores carencias. La actual estructura de subsidios al agua domiciliaria en CDMX — a través de descuentos en las tarifas establecidas-, intenta beneficiar a los hogares pobres con consumos bajos en metros cúbicos al aplicar un descuento de 90 por ciento sobre la tarifa popular; el subsidio baja a medida que la tarifa se incrementa hasta llegar a la tarifa alta en la que el subsidio es de 60 por ciento. Sin embargo, los resultados muestran que no se está alcanzando el objetivo planteado, y que esto se debe a que existen importantes fallas en la focalización de la posición socioeconómica del hogar y sus correspondientes tarifa y subsidio.

La escasa información proporcionda por Sacmex sigue siendo una restricción para este tipo de estudios, por lo que debe generarse a través de encuestas que son costosas y las exigencias metodológicas del diseño muestral tienen que restringirse al presupuesto del proyecto. Es importante anotar que las conclusiones se basan en una encuesta levantada de manera particular, que cumple con la rigurosidad metodológica estándar; sin embargo, una limitante de la información es que el diseño muestra se basó en un marco muestral de hogares del INEGI de 2008. La variable determinante para el diseño de la muestra fue el acceso al agua entubada, y una consecuencia de esta situación es que la variable ingresos del hogar no se pudo trabajar con un nivel de desagregación mayor, lo que permitiría compatibilidad con otras estadísticas nacionales e internacionales.

Se sugiere conjuntar sinergias en el desarrollo de proyectos entre los gobiernos interesados en el tema y las universidades para realizar las investigaciones con 
mayores recursos que permitan generar indicadores con el grado de detalle requerido para la toma de decisiones de política pública.

\section{Implicaciones}

A pesar de la popularidad del uso de la estructura de bloques incrementales (IвT) para el establecimiento de tarifas para el servicio de agua potable — como es el caso de CDMX-, existen principalmente dos razones para que los subsidios no sean efectivos para llegar a hogares de ingresos económicos bajos y medio bajos (Whittington et al., 2015). En primer lugar, sólo los hogares con conexiones al servicio de agua potable pueden recibir el subsidio a través de la tarifa. Sin embargo, en la mayoría de los países menos desarrollados o emergentes, por lo general los hogares con menores ingresos económicos no tienen conexiones directas al servicio o son conexiones deficientes o comunitarias (Whittington, et al., 2015; Barde y Lehmann, 2014). En segundo lugar, la carencia de información confiable para identificar y relacionar las variables socioeconómicas de los diferentes hogares con el consumo de agua potable puede ocasionar que hogares con ingresos económicos medios o altos puedan pasar por hogares con bajos ingresos.

En la CDMX se registran ambas situaciones, en el primer caso, existen $350 \mathrm{mil}$ hogares que aún no están conectadas al servicio público (INEGI, 2010, 2015) y son los hogares con los menores ingresos económicos y las mayores necesidades, y se abastecen por medio de carro-tanques, agua embotellada o pipas, que generalmente tienen a precios mucho más altos, el agua es de menor calidad y el servicio no es continuo; además, al no tener acceso al consumo de agua domiciliaria no reciben el subsidio. En el segundo caso, el hecho de no identificar de manera correcta el tipo de hogar con su respectiva clasificación socioeconómica ocasiona la aplicación ineficiente de los subsidios.

En la CDMX es prioritario dotar de agua potable a los hogares más pobres y vulnerables, es la manera más eficiente de canalizar subsidios a este grupo social. Una manera efectiva es destinar mayores niveles de inversión para este grupo y establecer subsidios a la conexión (Komives et al., 2005). En segundo lugar, se debe focalizar con mayor precisión a los hogares, en particular a los pobres, lo que permitiría canalizar bien los subsidios. Por ejemplo, Komives et al. (2005) plantean que la focalización de los subsidios genera tres beneficios para la sociedad; primero, permite la reducción del presupuesto de los subsidios o el costo de ofrecerlos; segundo, la focalización significa un mayor impacto sobre aquellos hogares pobres que más lo necesitan, al destinar una mayor proporción del pre- 
supuesto a este tipo de hogares, y tercero, los subsidios que se dirigen a menos hogares tienen el potencial de causar menores distorsiones respecto al consumo que si los subsidios están mal dirigidos.

En el caso de la aplicación de subsidios, éstos deben estar bien focalizados para reducir las condiciones de pobreza de los hogares, básicamente al ofrecer el servicio de agua corriente a dichos hogares y garantizar que los subsidios efectivamente lleguen a quienes los necesiten. Pero al mismo tiempo, la tarifa que se cobre al usuario del servicio no debe ser tan alta que impida acceder al recurso, ni tan baja que incentive el desperdicio de éste (Jiménez et al., 2011).

Recomendaciones de organismos internaciones como la Organización para la Cooperación y el Desarrollo Económicos (ocDE) o el Centro Panamericano de Ingeniería Sanitaria y Ciencias del Ambiente (CEPIS) indican que por lo menos se debe recuperar el costo de operación y mantenimiento por medio de las tarifas implementadas, situación que de no cumplirse puede ocasionar problemas financieros para los prestadores del servicio (OCDE, 1989; Cepis, sf). La experiencia de Colombia resulta sugerente para el Sacmex, que enfrenta actualmente un escenario de estrés financiero. En aquel país se ha desarrollado un sistema de focalización o estratificación de las viviendas en función del ingreso del hogar y de las características de construcción de la vivienda, así como del entorno en el cual está construida (Econometría, 2008). La estratificación de hogares identifica seis grupos, los estratos más bajos corresponde a aquellos donde habitan usuarios con menores recursos y con mayores necesidades, los cuales son beneficiarios de los subsidios de los servicios públicos domiciliarios; los estratos de mayores recursos pagan un sobrecosto sobre el valor de los servicios (subsidio cruzado). Y el estrato medio no es beneficiario de subsidios, paga exactamente el valor que la empresa prestadora del servicio establezca como costo de suministro. $\mathbf{G}$ 으

\section{REFERENCIAS BIBLIOGRÁFICAS}

ALDF (Asamblea Legislativa del Distrito Federal) (2011), "Decreto por el que se expide el Código Fiscal del Distrito Federal”, Gaceta del Distrito Federal, 29 de diciembre de 2009, disponible en: http://www.aldf.gob.mx/archivo-afa2182ed56e3aebca333d7b68feed36. pdf [fecha de consulta: 13 de junio de 2018].

Ángel-Urdinola, D.F. y Q. Wodon (2012), "Do Utility Subsidies Reach the Poor? Framework and Evidence for Cape Verde, Sao Tome, and Rwanda", Economics Bulletin, 9(4), pp. 1-7. Banco Mundial (1995), "Vietnam Poverty Assessment and Strategy", reporte 13442-VN, Country Department I, East Asia and Pacific Region, Washington D.C.: Banco Mundial. 
Banerjee, S., V. Foster, Y. Ying, H. Skilling y Q. Wodon (2010), "Cost Recovery, Equity and Efficiency in Water Tariffs: Evidence from African Utilities", document de trabajo 5384, Washington, D.C.: Banco Mundial.

Bardasi, E. y Q. Wodon (2008), "Who Pays the Most for Water? Alternative Providers and Service Costs in Niger", Economics Bulletin, 9(20), pp. 1-10.

Barde, J.A. y P. Lehmann (2014), "Distributional Effects of Water Tariff Reforms: An Empirical Study for Lima, Peru”, Water Resources and Economics, 6, pp. 30-57.

CePIs (Centro Panamericano de Ingeniería Sanitaria y Ciencias del Ambiente) (sf), Principios para el diseño de tarifas de agua y alcantarillado, disponible en: http://www.bvsde. paho.org/eswww/proyecto/repidisc/publica/hdt/hdt061.html [fecha de consulta: 20 de febrero de 2018].

Coneval (Consejo Nacional de Evaluación de la Política de Desarrollo Social) (2012), Informe de pobreza y evaluación en el Distrito Federal 2012, Ciudad de México: Coneval.

Cuenca, J.S. (2008), "Benefit Incidence Analysis of Public Spending on Education in the Philippines: A Methodological Note", documento de debate 09, Philippine Institute for Development Studies.

Demery, L. (2000), "Benefit Incidence: A Practitioner's Guide Poverty and Social”, Development Group, Washington, D.C.: Banco Mundial.

Econometría (2008), Evaluación de la estratificación socioeconómica como instrumento de clasificación de los usuarios y herramienta de asignación de subsidios y contribuciones a los servicios públicos domiciliarios, informe institucional, disponible en: https://www.dane. gov.co/files/geoestadistica/Evaluacion_Estratificaicon.pdf [fecha de consulta: 15 de marzo de 2018].

eHCSCA (Encuesta sobre Hábitos de Consumo, Servicio y Calidad del Agua de los Hogares del Distrito Federal ) (2011), Ciudad de México: UAm/ICyтdF.

ENIGH (Encuesta Nacional de Ingresos y Gastos de los Hogares) (2010), Módulo de Condiciones Socioeconómicas (ENIGH-MCS), Ciudad de México: INEGI.

ENIGH (Encuesta Nacional de Ingresos y Gastos de los Hogares) (2012), Módulo de Condiciones Socioeconómicas (ENIGH-MCS), Ciudad de México: INEGI.

ENIGH (Encuesta Nacional de Ingresos y Gastos de los Hogares) (2014), Módulo de Condiciones Socioeconómicas (eNIGH-MCS), Ciudad de México: INEGI.

Espinosa-García, A.C., C. Díaz-Ávalos, F.J. González-Villarreal, R. Val-Segura, V. Malváez Orozco y M. Mazzari-Hiriart (2015), "Drinking Water Quality in a Mexico City University Community: Perception and Preferences", EcoHealth, 12(1), pp. 88-97.

Foster, V. (2004), "Toward a Social Policy for Argentina's Infrastructure Sectors: Evaluating the Past and Exploring the Future", documento de trabajo 3422, Washington, D.C.: Banco Mundial. 
Foster, V. y C. Araujo (2004), "Does Infrastructure Reform Work for the Poor? A Case Study from Guatemala”, documento de trabajo 3185, Washington, D.C.: Banco Mundial.

Galiani, S., P.J. Gertler y E. Schargrodsky (2005), "Water for Life: The Impact of the Privatization of Water Services on Child Mortality”, Journal of Political Economy, 113, pp. 83-120.

Gasparini, L., M. Cicowiez y W. Sosa Escudero (2012), "Pobreza y desigualdad en América Latina: Conceptos, herramientas y aplicaciones", documento de trabajo 171, La Plata: Universidad Nacional de La Plata-Centro de Estudios Distributivos, Laborales y Sociales (Cedlas).

GCDMx (Gobierno de la Ciudad de México) (2012), "Informe de avance trimestral enerodiciembre 2012", disponible en: https://data.finanzas.cdmx.gob.mx/documentos/ IAT_ene_dic_2012.pdf [fecha de consulta: 15 de junio de 2018].

GCDMx (Gobierno de la Ciudad de México) (2016), "Informe de avance trimestral enero-marzo 2016", disponible en: https://data.finanzas.cdmx.gob.mx/documentos/ banco_info_2016_1/Informe_Ene_Mar_2016.pdf [fecha de consulta: 15 de junio de 2018].

INEGI (Instituto Nacional de Estadística y Geografía) (2010), Censo de Población y Vivienda 2010, Ciudad de México: INEgr.

INEGI (Instituto Nacional de Estadística y Geografía) (2015), Encuesta Intercensal de Población y Vivienda 2015, Ciudad de México: INEGI.

Jiménez, B., R. Gutiérrez y B. Marañón (2011), “Evaluación de la política de acceso al agua potable en el Distrito Federal, México", Ciudad de México: unam.

JGDF (Jefatura de Gobierno del Distrito Federal) (2011), "Decreto por el que se expide la Ley de Ingresos del Distrito Federal para el ejercicio fiscal 2012”, Gaceta Oficial del Distrito Federal, 29 de diciembre de 2011, disponible en: https://data.finanzas.cdmx. gob.mx/documentos/LeyIngresos_2012.pdf [fecha de consulta: 21 de junio de 2018]. JGDF (Jefatura de Gobierno del Distrito Federal) (2012), "Decreto por el que se expide la Ley de Ingresos del Distrito Federal para el ejercicio fiscal 2013”, Gaceta Oficial del Distrito Federal, 31 de diciembre de 2012, disponible en: https://data.finanzas.cdmx. gob.mx/documentos/LeyIngresos_2013.pdf [fecha de consulta: 21 de junio de 2018]. Kochhar, K., C.A. Pattillo, Y. Sun, N. Suphaphiphat, A. Swiston, R. Tchaidze, B.J. Clements, S. Fabrizio, V. Flamini, L. Redifer y H. Finger (2015), "Is the Glass Half Empty or Half Full? Issues in Managing Water Challenges and Policy Instruments", notas de discusión 15/11, Washington D.C.: Fondo Monetario Internacional.

Kelly, P., S. Hicks, J. Oloya, J. Mwansa, L. Silkakwa, I. Zulu y A. Phillips (2003), "Escherichia coli Enterovirulent Phenotypes in Zambians with AIDs-Related Diarrhea", Transcripts of the Royal Society for Tropical Medicine and Hygiene, 97(5), pp. 573-576. 
Komives, K., J. Halpern, V. Foster, Q. Wodon y R. Abdullah (2005), Water, Electricity, and the Poor: Who Benefits from Utility Subsidies?, Washington, D.C.: Banco Mundial.

Komives, K., J. Halpern, V. Foster, Q. Wodon y R. Abdullah (2006), “The Distributional Incidence of Residential Water and Electricity Subsidies”, documento de investigación 3878, Washington, D.C.: Banco Mundial.

Komives, K., J. Halpern, V. Foster, Q. Wodon y R. Abdullah (2007), “Utility Subsidies as Social Transfers: An Empirical Evaluation of Targeting Performance”, Development Policy Review, 25(6), pp. 659-679.

LNa (Ley Nacional del Agua) (2016), Cámara de Diputados del H. Congreso de la Unión, Ciudad de México, 24 de marzo, disponible en: http://www.diputados.gob.mx/LeyesBiblio/pdf/16_240316.pdf [fecha de consulta: 30 de marzo de 2018].

Listori, J.A. (1996), "Bridging Environmental Health Gaps: Lessons for Sub-Saharan Africa Infrastructure Projects: Main Report”, Africa Technical Department Environmentally Sustainable Development Division (Aftes), documentos de trabajo 20, 21 y 22, Washington, D.C.: Banco Mundial.

Mazari-Hiriart, M., S. López-Vidal, S. Ponce de León, J.J. Calva, F. Rojo-Callejas y G. Castillo-Rojas (2005), "Longitudinal Study of Microbial Diversity and Seasonality in the Mexico City Metropolitan Area Water Supply System”, Applied and Environmental Microbiology, 71(9), pp. 5129-5137.

Morales-Novelo, J. y L. Rodríguez-Tapia (2007), “Economía del agua: Escasez del agua y su demanda doméstica e industrial en áreas urbanas", Ciudad de México: Cámara de Diputados-LX Legislatura/UAM.

Nina, E. (2011), "Efecto del gasto público social en la distribución del ingreso en Colombia”, Rúbricas, otoño-invierno, pp. 58-65.

ocDE (Organización para la Cooperación y el Desarrollo Económicos) (1989), Water Resources Management: Integrated Policies, París: OCDE.

Perló, M. y A.E. González (2005), ¿Guerra por el agua en el Valle de México?, Ciudad de México: Universidad Nacional Autónoma de México/Fundación Friedrich Ebert.

Rodríguez-Tapia, L., D.A. Revollo-Fernández y J.A. Morales-Novelo (2016), "Household's Perception of Water Quality and Willingness to Pay for Clean Water in Mexico City", Economies, 5(2), pp. 1-14.

Sen, A. (1995), “The Political Economy of Targeting”, en D. van de Walle y K. Nead (eds.), Public Spending and the Poor: Theory and Evidence, Washington, D.C.: Banco Mundial, pp. 11-24.

Stiglitz, Joseph E. (2000), La economía del sector público, Barcelona: Antoni Bosch. Soto-Montes de Oca, G. (2015), "Tarifas y derecho humano al agua”, Revista de Derechos Humanos, 7, pp. 21-27. 
Subbarao, K., A. Bannerjee, K. Ezemerari, J. Braithwaite, C. Graham, S. Carvalho y A. Thompson (1997), Safety Net Programs and Poverty Reduction: Lessons from Cross-Country Experience, Washington. D.C.: Banco Mundial.

Tortajada, C. (2006), "Water Management in Mexico City Metropolitan Area", International Journal of Water Resources Development, 22(2), pp. 353-376.

Vélez, C. (1996), Gasto público social y desigualdad: Logros y extravios, Bogotá: Departamento Nacional de Planeación.

Walker, L., P. Ordóńez, P. Serrano y J. Halpern (2000), "Pricing Subsidies and the Poor: Demand for Improved Water Services in Central America”, World Bank Policy Research Paper 2468, Washington, D.C.: Banco Mundial.

Whittington, D., C. Nauges, D. Fuente y X. Wu (2015), "A Diagnostic Tool for Estimating the Incidence of Subsidies Delivered by Water Utilities in Low — and Medium- Income Countries, with Illustrative Simulations", Utilities Policy, 34, pp. 70-81.

Daniel A. Revollo-Fernández tiene un posdoctorado en Valoración Económica de Bienes y Servicios Ambientales en el Centro Regional de Investigaciones Multidisciplinarias (CRIM-UNAM), es doctor en Economía de Recursos Naturales y Desarrollo Sustentable de la Universidad Nacional Autónoma de México, magister en Economía de la Universidad de los Andes (Colombia) - University of Maryland at College Park (Estados Unidos) y licenciado en Economía de la Universidad Católica de Bolivia (Bolivia). Es miembro del Sistema Nacional de Investigadores (sNI), nivel I. Tiene intereses en temas de economía ambiental, técnicas de valoración económica ambiental, teoría de juegos, economía experimental en manejo de recursos de uso común, cambio climático y políticas publicas relacionadas con transporte, agua y alcantarillado. Fue asesor de la Subdirección Técnica de la Comisión de Regulación de Agua Potable y Saneamiento Básico del Ministerio de Ambiente, Vivienda y Desarrollo Territorial de Colombia. Profesor en diversas universidades de materias como econometría, microeconomía, valoración económica ambiental y economía de recursos naturales.

Lilia Rodríguez-Tapia es licenciada en Economía por la Universidad Autónoma Metropolita-Azcapotzalco y licenciada en Relaciones Internacionales con especialidad en Comercio Internacional por el Instituto Politécnico Nacional. Maestra en Economía por el CIDE y doctora en Ciencias Económicas por la UAM. Es miembro del Sistema Nacional de Investigadores (sNI), nivel I. Profesor perfil Promep. Coordinadora del Cuerpo Académico Consolidado Promep. "Gestión Integral del Sistema Natural y Económico" clave UAM-CA-120, cuerpo académico vigente del 18 de noviembre de 2010 al 18 de 
noviembre de 2015. Miembro de la Red Temática del Agua (Retac) Conacyt desde 2009. Miembro del Comité Mexicano para la Atención de la Organización Internacional de Estandarización (ISO) desde 2008. Miembro del Comité de la Red Metropolitana del Agua, uAM, desde 2006. Ha impartido los cursos de Economía Ambiental I y II, Precálculo y Cálculo Diferencial e Integral en la licenciatura en Economía; así como los cursos de Socioeconomía Ambiental, Temas Selectos de Ciencias e Ingeniería Ambientales I y II, Proyecto de Investigación en Ambiental I, II y III en el posgrado en Ciencias e Ingeniería Ambientales, UAM-A. Asimismo, ha impartido la materia de Valoración Económica del Ambiente para el diplomado en Política, Derecho y Gestión Ambiental. Ha publicado artículos en revistas nacionales e internacionales, libros y capítulos de libros sobre la relación de la economía y el agua, además de haber participado en numerosos eventos científicos nacionales e internacionales. También ha dirigido tesis de licenciatura y posgrado.

Jorge A. Morales-Novelo es licenciado en Economía por la Universidad Nacional Autónoma de México y doctor en Ciencias Económicas por la Universidad Autónoma Metropolitana. Miembro fundador del área de investigación Crecimiento Económico y Medio Ambiente, Departamento de Economía, Universidad Autónoma Metropolitana-Unidad Azcapotzalco. Miembro del Sistema Nacional de Investigadores (SNI), nivel I. Profesor perfil Promep. Miembro del Cuerpo Académico Consolidado Promep "Gestión Integral del Sistema Natural y Económico" clave UAM-CA-120, vigente desde el 18 de noviembre de 2010 al 18 de noviembre de 2015. Miembro de la Red Temática del Agua (Retac) Conacyt desde 2009. Miembro del Comité de la Red Metropolitana del Agua, uam desde 2006. Miembro de la Red Temática del Agua de Conacyt (Retac) desde 2009. Miembro del Comité Mexicano para la Atención de la Organización Internacional de Estandarización (Iso) desde 2008. Imparte cursos de Economía Ambiental I y II, Teorías del Crecimiento Económico I y II en la licenciatura en Economía y el curso de Socioeconomía Ambiental en el posgrado en Ciencias e Ingeniería Ambientales. Ha publicado artículos en revistas nacionales e internacionales, libros y capítulos de libros sobre la relación de la economía y el agua; además de haber participado en diversos eventos científicos nacionales e internacionales. Asimismo, ha dirigido tesis de licenciatura y posgrado. 